\title{
ROLE OF TUMOUR SUPPRESSOR GENE P53 IN TRIPLE NEGATIVE BREAST CANCER
}

\author{
Priya S Patil, Jaydeep N Pol, Ashalata D Patil.
}

${ }^{{ }^{*}}$ Associate Professor, Bharati Vidyapeeth Deemed University Medical College \& Hospital, Sangli, Maharashtra, India.

${ }^{2}$ Consultant Pathologist, Mahatma Gandhi Oncopathology Center, Miraj, Maharashtra, India.

${ }^{3}$ Professor, Department of Anatomy, DY Patil Medical College, Kolhapur, Maharashtra, India.

\section{ABSTRACT}

Background: Cancer is a rapidly growing health problem in both developed and developing countries. In the diagnosis and prognosis of breast cancer tumour markers are routinely used. However $10-20 \%$ of breast cancers test negative for hormone receptors [ER, PR] as well as HER2 and are labelled as Triple-negative breast cancers (TNBC). TNBCs' show a higher histological grade, metastasize and recur after treatment with lower five-year survival rates. Cancer genetics is the study of oncogenes and tumour suppressor genes. The tumour suppressor gene p53 called as the guardian of the genome plays its own role in such cancers. When mutation occurs in p53 it affects multiple oncogenic processes. Current study focuses on triple negative breast cancers and the study of cancer critical tumour suppressor gene p53 in TNBC by immunohistochemistry.

Materials and Methods: In collaboration with oncopathology center the study was conducted in the department of Anatomy for a period of two years. All the cases of carcinoma breast were included and retrospectively reviewed for histological grade, hormone and HER2 status. The TNBCs' were stained further for p53 by Immunohistochemistry in the department of Anatomy.

Results and Conclusion: In the current study the incidence of TNBCs' was $23 \%$. Out of total TNBCs' $27 \%$ were p53 positive and all of them were diagnosed at an earlier age. The histological grade of all p53 positive TNBCs' was grade III which showed statistical significance. The p53 positive TNBCs' definitely need aggressive treatment with close follow up.

KEY WORDS: Tumour suppressor gene p53, triple negative breast cancers, TNBC.

Address for Correspondence: Dr. Priya S Patil, Associate Professor, Bharati Vidyapeeth Deemed University Medical College \& Hospital, Sangli, Maharashtra, India.

E-Mail: drpriyapatil22@gmail.com

\section{Access this Article online}

\section{Quick Response code}

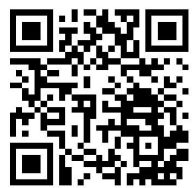

DOI: $10.16965 /$ ijar.2017.402

Web site: International Journal of Anatomy and Research ISSN 2321-4287

www.ijmhr.org/ijar.htm

Received: 08 Aug 2017

Peer Review: 09 Aug 2017

Revised: None
Accepted: 03 Oct 2017

Published (O): 01 Nov 2017

Published (P): 01 Nov 2017

\section{INTRODUCTION}

Cancer research currently focuses on new molecular markers which would help to identify cancer critical genes. The combination of such molecular markers into existing classification of cancers will provide more accurate diagnosis as well as prognosis [1].

To enhance this and provide molecular defini tions of specific cancers like breast cancers, many advanced diagnostics are being utilized from histopathology to molecular histology methodslike immunohistochemistry, microarrays,comparative genomic hybridization and others. Immunohistochemistry allows detection of tumour markers in breast cancer like the hormone receptors which receive messages 
from the hormones oestrogen and progesterone or receptors for the gene HER2 (human epidermal growth factor receptor 2). Hormone receptors control the cell's behaviour and are done routinely to know the status of the tumour as well as a guide to the prognosis. However not all breast cancers have these receptors and about 10-20\% of breast cancers test negative for both hormone receptors and HER2 and are labelled as Triple-negative breast cancers (TNBC). These TNBCs' are currently gaining increasing interest in research and researchers are working to find new and better ways to treat it [2].

Due to the complex molecular patterns involved in TNBCs', the search for novel biomarkers and therapy targets requires collection of multidimensional data analysis [2]. In this scenario p53 gene which is a tumour suppressor gene plays a vital role. Wild type p53 protein can suppress cellular transformation and proliferation by regulating cell cycle at $\mathrm{G} 1 / \mathrm{S}$ phase as well as induce apoptosis following DNA damage [3]. Mutation in tumour suppressor gene leads to cancer where cell division loses all normal restraints. Such p53 mutations are seen to be present in more than $50 \%$ of all tumours. ${ }^{[4]}$ The role of mutant p53 in cancer initiation and progression can be very useful in solid tumour profiling, predicting the course of the disease, resistance to routinely used first line drugs, prognosis, monitoring and recurrence of disease [4]. Considering the individual impact of TNBC and p53 status their combined study can provide new insights to the clinician in understanding breast cancers. Current research was focused on triple negative breast cancers and the study of cancer critical tumour suppressor gene $\mathrm{p} 53$ in them by immunohistochemical staining. Further we have highlighted the possible outcome in such a scenario and future avenues for research.

\section{MATERIALS AND METHODS}

After the consent and approval from the concerned institutes the study was conducted in the department of Anatomy in collaboration with an oncopathology centre. It was a retrospective study for a period of two years from January 2013 to December 2014. All the cases of breast cancers diagnosed at the Oncology centre and sent to their laboratory for histopathology during this period were included in the study. The Paraffin blocks of the cases and the clinical files of the patients were reviewed. The Haematoxylin - Eosin stained [ $H \& E$ ] slides were used for histopathological reporting. The histological grading of the tumours done by the pathologist was obtained from their previous records.

Immunohistochemistry [IHC]-The IHC reports on $E R, P R$ and HER2 status were reviewed from the previous records. The cases were separated on basis of receptor status on Immunohistochemistry and the blocks of triple negative breast cancer cases were isolated. These blocks were taken to the Department of Anatomy and were further processed there and used for $\mathrm{p} 53$ staining.

Immunohistochemistry [IHC]- P53 staining protocol was followed according to manufacturer's instructions. [DAKO Kits]

The blocks were sectioned to obtain 5-7 u sections which were fixed on pre-coated slides. The sections were deparaffinised and hydrated to water. Peroxidase blocking was performed with $3 \% \mathrm{H} 2 \mathrm{O} 2$ followed by three washings with PBS. Antigen retrieval was done with citrate buffer [pH 6] in microwave two times at $900 \mathrm{~W}$ for 10 minutes each and one time at $720 \mathrm{~W}$ for 10 minutes. The sections were cooled to room temperature followed by washings with PBS. Power block was done for 20 minutes. The sections were treated with primary antibody against $\mathrm{p} 53$ for 60 minutes in humid chamber followed by three washings with PBS. They were further treated with polymer horse radish peroxidase HRP for 45 minutes followed by washings with PBS. This was followed by DAB chromogen for 10 minutes, then stained by Haematoxylin for 2 minutes, followed by water wash followed by Acid alcohol dip and bluing for 5 minutes. The slides were then dried, mounted and observed under microscope and reviewed for $\mathrm{p} 53$ staining.

\section{RESULTS}

In the study period of two years a total of 213 cases of carcinoma of breast were obtained for the study shown in Table 1.

The Haematoxylin \& Eosin stained slides were observed and assessed for histological grade of tumour. On Immunohistochemistry they were 
further assessed for ER, PR and HER2 status. Those cases which were triple negative were isolated and selected for further study. Out of 213 cases 50 were triple negative. The incidence of TNBC was $23.47 \%$ in the current study. The histological type and grading were noted from the previous histopathological reports. Of 50 TNBC 49 were infiltrating duct carcinomas while only one was lobular type.

All the 50 triple negative cases were further stained for p53 status. Due to technical errors and staining limitations 41 blocks could be stained up to the mark and were included in the study. Of the 41 cases 11 cases were positive for $p 53$.All the slides were frankly positive and showed accumulation of DAB chromogen [brown colour] in the nuclei of the clusters of malignant ductal cells.[Figures 1,2 ] The p53 positivity in TNBC was $26.8 \%$ in the current study.

Correlation of $p 53$ with age of patient: The age of all the patients having carcinoma breast ranged between 30 to 71 years, mean age being 51.9 years with a standard deviation of 10.6 years. The age of $p 53$ positive and $p 53$ negative patients are shown in Table 2 . p53 positive patients showed a mean age of 47 years which was on a lower side as compared to the mean age of p53 negative patients. However the p value as calculated by the unpaired test was 0.06 and was not statistically significant.

Correlation of p53 status with tumour grade is shown in the Graph 1.The p53 positive tumours showed $100 \%$ grade III lesions on histology while in p53 negative tumours $70 \%$ were of grade III and $30 \%$ grade II. The $p$ value was calculated using Fisher's exact test and was 0.04 which was statistically significant.

Table 1: Distribution of cases of cancer breast and TNBCs'.

\begin{tabular}{|c|c|c|c|}
\hline Sr.no & Year & $\begin{array}{c}\text { Total cases of Ca } \\
\text { breast }\end{array}$ & TNBC \\
\hline 1 & Jan 2013 to Dec 2013[12 months] & 95 & 23 \\
\hline 2 & Jan 2014 to Dec 2014[12 months] & 118 & 27 \\
\hline Total & 2 years & 213 & 50 \\
\hline
\end{tabular}

Table 2: Age of patients with p53 positive TNBC.

\begin{tabular}{|c|c|c|c|c|c|}
\hline P53 & N & Mean & $\begin{array}{c}\text { Std. } \\
\text { Deviation }\end{array}$ & $\begin{array}{c}\text { Std. Error } \\
\text { Mean }\end{array}$ & p value \\
\hline Positive & 11 & 47.09 & 9.02 & 2.72 & \multirow{2}{*}{0.06} \\
\hline Negative & 30 & 53.7 & 10.71 & 1.96 & \\
\hline
\end{tabular}

Graph 1: Histological grade of p53 negative and positive breast cancers.

\section{HISTOLOGICAL GRADE OF BREAST CANCERS}

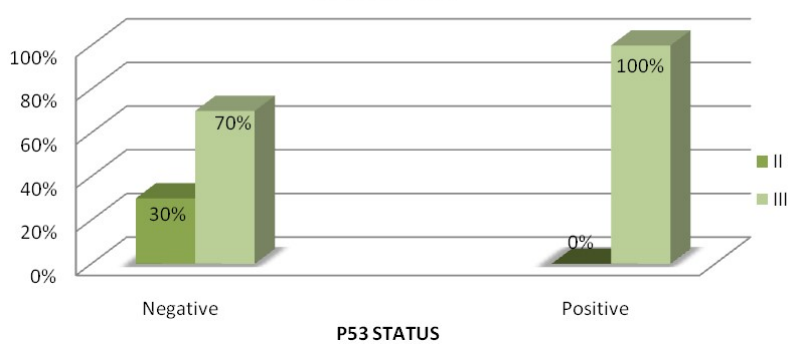

Fig. 1: Immunohistcemisty for p53 showing positve staining with DAB chromogen accumulated in the nuclei of cancer cells.

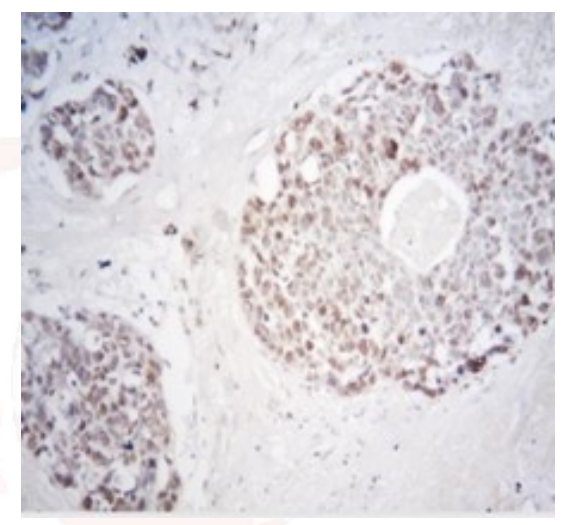

Fig. 2: Immunohistcemisty for p53 showing positve nuclear staining with $D A B$ chromogen.

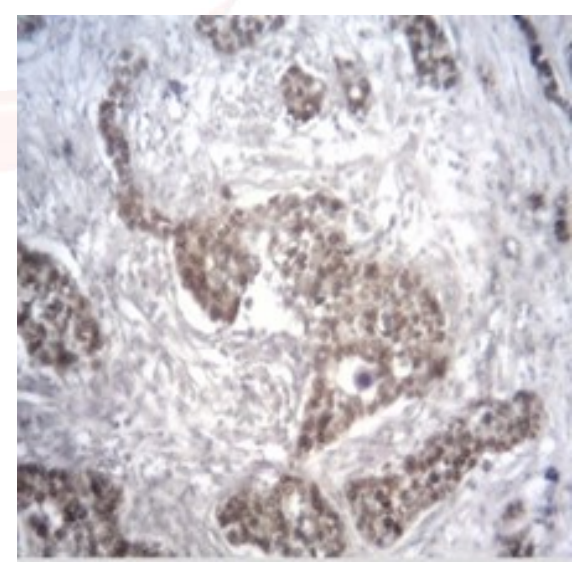

\section{DISCUSSION}

The overall incidence of cancer as well as breast cancer is a rapidly growing health problem in both developed as well as developing countries. Cancer critical genes can be classified into two groups - one having gain of function mutation that converts proto-oncogenes to oncogenes and stimulate cells to increase in number when they should not and a loss of function mutation of tumour suppressor genes which abolish the inhibitory controls which hold the cell number in check. Cancer genetics refers to the study of 
oncogenes and tumour suppressor genes which can now be identified by sophisticated diagnostic methods [1].

The tumour suppressor gene $p 53$ was first described in 1979. It lies on the short arm of chromosome 17 and encodes a nuclear phosphoprotein. Wild type $\mathrm{p} 53$ is also called as the guardian or friend of the genome as it can suppress cellular transformation and proliferation by regulating cell cycle at $\mathrm{G} 1 / \mathrm{S}$ phase [4]. It encourages repair of the damaged DNA and if that is not possible acts by inducing apoptosis following DNA damage through proapoptotic proteins. Its major role is that it acts as an inhibitor of angiogenesis and metastasis [4].

Mutation in p53 tumour suppressor gene converts this friend to a foe. According to literature, mutation in p53 gene is found in 30 to 50 $\%$ of all and $23 \%$ of breast cancers where the cells become immortal and proliferate $[4,5]$. The mutant p53 plays a vital role in breast cancers through various mechanisms. There can be germ line mutations leading to the Li-Fraumeni cancer susceptibility syndrome or somatic mutations such as loss of function mutation, frame shift mutation or missense mutation $[4,5]$. This leads to inactivation of the $\mathrm{p} 53$ pathway and to the stable expression of the mutant p53 in the nucleus of cancer cells as against wild type p53 which has a very short half life. This mutant p53 is responsible for more aggressive tumour profile due to acquisition of some novel functions which promote tumorigenesis [5,6]. In a study by Olivier $\mathrm{M}$ et al [7] p53 mutations were more frequent in high grade, large size, node positive breast cancers with negative ER, PR receptors. Such tumours which are ER, PR negative as well as negative for HER2 are called triple negative breast cancers TNBC. In our study too p53 mutations are seen in all high grade tumours with statistical significance.

The incidence of TNBCs' according to literature review is around 10 to $20 \%$ of total breast cancers [3]. In our study the incidence of TNBC is $23.47 \%$ which correlates with findings of other researchers. Studies have shown that a TNBC is more likely to spread beyond the breast so also more likely to recur after treatment. They tend to be of a higher histological grade than other types of breast cancers and five-year sur- vival rates tend to be lower for a TNBCs' [8] Further follow up of these TNBCs' would be of prime importance to throw light on disease progression and five year survival. Though recent evidences suggest significant advances in treatment of patients with breast carcinomas, a targeted therapy for TNBC is still elusive [8].

According to a study in 2014 by Naoise C, et al [9] more than $80 \%$ of TNBC have p53 mutations. However in the current study out of total TNBCs' $26.8 \%$ were $p 53$ positive and all of them were diagnosed at an earlier age. This incidence is similar to p53 positivity in all breast cancers which was about $23 \%$ [5].

Presence of $\mathrm{p} 53$ mutation in an already aggressive hormone negative tumour further worsens the disease and its prognosis. The mutant p53 affects multiple oncogenic processes in early tumorigenesis due to genomic instability and antiapoptotic effect. Mutant p53 also has the ability to sustain tumour growth and promote vascular endothelial growth factor (VEGF) expression leading to angiogenesis. The increased incidence of metastasis in p53 positive tumours is contributed to epithelialmesenchymal transition of mammary epithelial cells. This generates cancer stem-like cells with mesenchymal properties of invasion and motility. They penetrate vascular endothelium resulting in distant metastasis. This correlates with the poor patient survival in such cases [5]. Presence of p53 mutations in breast cancers especially in a TNBC therefore affects disease free survival with increased rate of recurrence and visceral metastasis [10].

The recent evidences suggest that most mutant p53s are expressed at high levels in cancer cells and can be detected immunohistochemically, so p53 proteins serve as attractive therapeutic targets that hold great promise in future [10]. Hence isolating TNBCs' and knowing their p53 status would definitely offer a paradigm shift in the outlook of the clinician. Such a diagnosis of p53 positive TNBC would alert the clinician to change his approach to a more aggressive line and newer drugs instead of the traditional treatment protocol. Newer compounds that bind to multiple mutant $\mathrm{p} 53$ proteins e.g., PRIMA-1 or PRIMA-met, may help in restoration of p53 function. ${ }^{[11]} A$ number of hurdles still need to be 
overcome. Larger prospective clinical trials with follow up of TNBCs' with p53 positivity are needed to optimize treatments and predict their response in patients of breast cancers.

\section{CONCLUSION}

TNBC $s^{\prime}$ and their course are known for their adamant behaviour. In addition to this a mutant p53 in such TNBCs' further mesmerizes the scenario. In such a scenario p53 status can serve as a good guide as it divides TNBCs' into two biologically distinct subgroups as p53 positive TNBC and p53 negative TNBC. Of these the p53 positive TNBCs' definitely need aggressive and targeted treatment with a close follow up.

\section{ACKNOWLEDGEMENTS}

We sincerely thank V.C, D.Y.Patil,University for funding this project. We extend gratitude to MGM Hospital staff and patients for their cooperation during the project. A sincere thanks to all the colleagues, technicians and statistician for their support.

\section{Conflicts of Interests: None}

\section{REFERENCES}

[1]. Bruce Alberts, Alexander Johnson, Julian Lewis, Martin Raff, Kith Roberts, Peter Walter. Cancer, in Molecular Biology of the Cell $5^{\text {th }}$ edn: USA; Garland Science, Taylor and Francis group, 2008:234-50.

[2]. Daniel Dang, Yang Peng. Roles of p53 and p6 in triple- negative breast cancer. Breast Cancer Manage 2013;2(6):537-544.
[3]. Lane DP: P53; Guardian of the genome. Nature 358:1992,p:15-16.

[4]. Milena Gasco,Shukri Shami, Tim Crook. Review: The p53 pathway in breast cancer. Breast Cancer Res, 2002;4:70-76.

[5]. Walerych D, Napoli M, Collavin L, Del Sal G. The rebel angel: mutant $\mathrm{p} 53$ as the driving oncogene in breast cancer. Carcinogenesis. 2012 Nov;33(11): 2007-17.

[6]. Patricia A.J. Muller, Karen H. Vousden. Mutant p53 in Cancer: New Functions and Therapeutic Opportunities. Cancer Cell. 2014 Mar 17;25(3):304-317.

[7]. Oliver $M$ etal.The clinical value of somatic TP53 gene mutations in 1,794 patients with breast cancer. Clin.Cancer Res.,12:1157-67.

[8]. Franca Podoa, Lutgarde M.C. Buydensb , HadassaDeganic , RietHilhorstd, EddaKlippe, Ingrid S. Gribbestadf, et al.Review Triple-negative breast cancer: Present challenges and new perspectives. Molecular Oncology,2010;4:209 -229.

[9]. Naoise C Synnott, Alyson M. Murray, Norma O'Donovan, John Crown and Michael J. Duffy. Mutant p53 as a therapeutic target for the treatment of triple-negative breast cancer: Prelinical investigation with the anti-p53 drug, APR-246. Journal of Clinical Oncology,2016;34(15):1082.

[10]. P.Yang, C.W.Du, M Kwan, S X Liang, G J Zhang. The impact of p53 in predicting clinical outcome of breast cancer patients with visceral metastasis. Scientific Reports,2013;3:1-6.

[11]. Jeremy M.R. Lambert, PetrGorzov, Dimitry B. Veprintsev, MajaSöderqvist, Dan Segerbäck, Jan Bergman, Alan R. Fersht, Pierre Hainaut, Klas G. Wiman, Vladimir J.N. Bykov. PRIMA-1 Reactivates Mutant p53 by Covalent Binding to the Core Domain. Cancer Cell 2009;15(5):376-388.

How to cite this article:
Priya S Patil, Jaydeep N Pol, Ashalata D Patil. ROLE OF TUMOUR
SUPPRESSOR GENE P53 IN TRIPLE NEGATIVE BREAST CANCER.
Int J Anat Res 2017;5(4.2):4585-4589. DOI: $10.16965 /$ ijar.2017.402 